\title{
«Robotik wird sich durchsetzen, wo sie Patienten Vorteile bringt»
}

\section{Das Interview führte Sabina Heuss}

Dr. phil., Fachhochschule Nordwestschweiz, Institute for Competitiveness and Communication

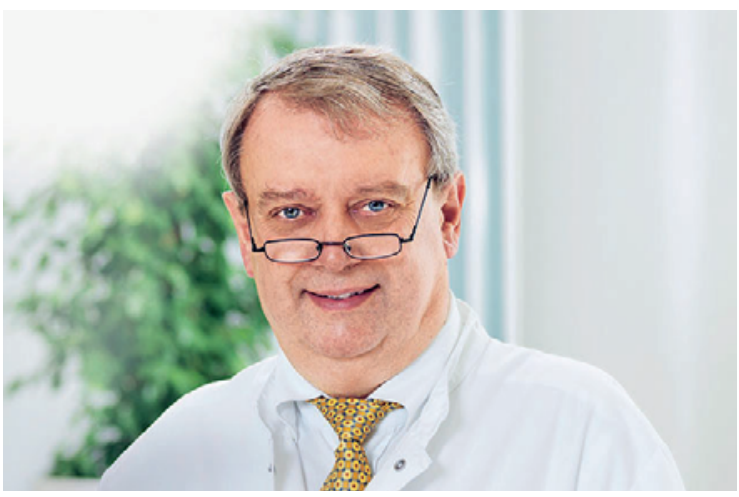

Prof. Hans-Florian Zeilhofer, Senior Consultant und Head of Research an der Klinik für Mund-, Kiefer- und Gesichtschirurgie des Universitätsspitals Basel, begleitet die Entwicklung der Robotik in der Medizin an vorderster Front. Er ist Leiter des HightechForschungszentrums des Department of Biomedical Engineering und Delegierter für Innovation der Universität Basel sowie CoFounder des Schweizer Startups Advanced Osteotomy Tools (AOT), das den Operationsroboter Carlo (Cold Ablation Robotguided Laser Osteotome) auf den Markt brachte.

\section{Herr Zeilhofer, wo stehen wir heute mit Robotern im Operationssaal?}

Hans-Florian Zeilhofer: Es ist richtig, dass Roboter heute bereits ein fester Bestandteil im Operationssaal sind. In der Regel sind diese Roboter, wie beispielsweise der Da-Vinci, nicht autonom. Das heisst, sie können nicht selbständig agieren. Sie werden vom Chirurgen über Telemanipulation geführt. Wir in Basel arbeiten in erster Linie an der Entwicklung von Robotern, die teilweise oder vollständig autonom bestimmte Aufgaben innerhalb einer Operation übernehmen können. Das lässt sich mit einem autonom fahrenden Auto vergleichen, welches von A nach B fährt. Dazu benötigt es ein GPS-System für die Streckenfestlegung sowie eine Sensorik, um Hindernisse zu erkennen und zu umfahren. Dasselbe gilt für den autonomen Operationsroboter. Der Chirurgieroboter Carlo (Cold Ablation Robot-guided Laser Osteotome), der seit diesem Jahr im Universitätsspital Basel zu präklinischen Forschungszwecken eingesetzt wird und an dessen Entwicklung ich beteiligt war, kann in beliebi- gen geometrischen Mustern berührungsfrei Knochen abtragen oder schneiden. In der Zukunft, mit entsprechender Sensorik und Künstlicher Intelligenz ausgestattet, wird er selbständig und in Echtzeit krankes von gesundem Gewebe unterscheiden können. Damit wird er unmittelbar Einfluss auf die Durchführung der Operation nehmen können.

\section{Die Entwicklung von Robotern geht ja oft von der Prämisse aus, dass damit der Arzt mehr Zeit für die Patientin hat. Stimmt das überhaupt?}

Der Roboter sollte mir als Arzt Aufgaben abnehmen und mich so weit entlasten, dass ich wieder mehr Zeit beim Patienten und beim Teachen der Kollegen in Ausbildung verbringen kann. Das wäre mein Wunsch. Leider hat die Digitalisierung im Gesundheitswesen uns immer wieder vor Augen geführt, dass wir zwar zeitsparende intelligente Softwaresysteme nicht mehr missen möchten, aber insgesamt zu viel Zeit für administrative Aufgaben am Computer aufwenden, die uns am Patienten letztendlich fehlt. Bei der Entwicklung von Medizinrobotern sollten wir diese alten Fehler nicht wiederholen. Das heisst, dass wir Ärzte, nicht die Gesundheitsökonomen, festlegen, wie viel «Arztzeit» unser Patient benötigt.

\section{«Wir arbeiten an der Entwicklung von Robo-} tern, die autonom bestimmte Aufgaben innerhalb einer Operation übernehmen können.»

\section{Der Roboter soll also die administrativen Aufgaben verringern?}

Natürlich, dazu sollte man den Roboter und seine digitale Operationsumgebung befähigen. Nach einer Operation könnte ein intelligentes System bereits den Rapport und den Arztbericht schreiben, Telefonate antizipieren und Materialien aus dem Lager nachbestellen. Das System könnte aber noch viel mehr: zum Beispiel Outcomes und Operationsverfahren mehrerer Spitäler oder Standorte vergleichen. Der Weg dorthin ist jedoch steinig. Hindernisse sind die nicht ausreichende Vernetzung unterschiedlicher Technologien, 


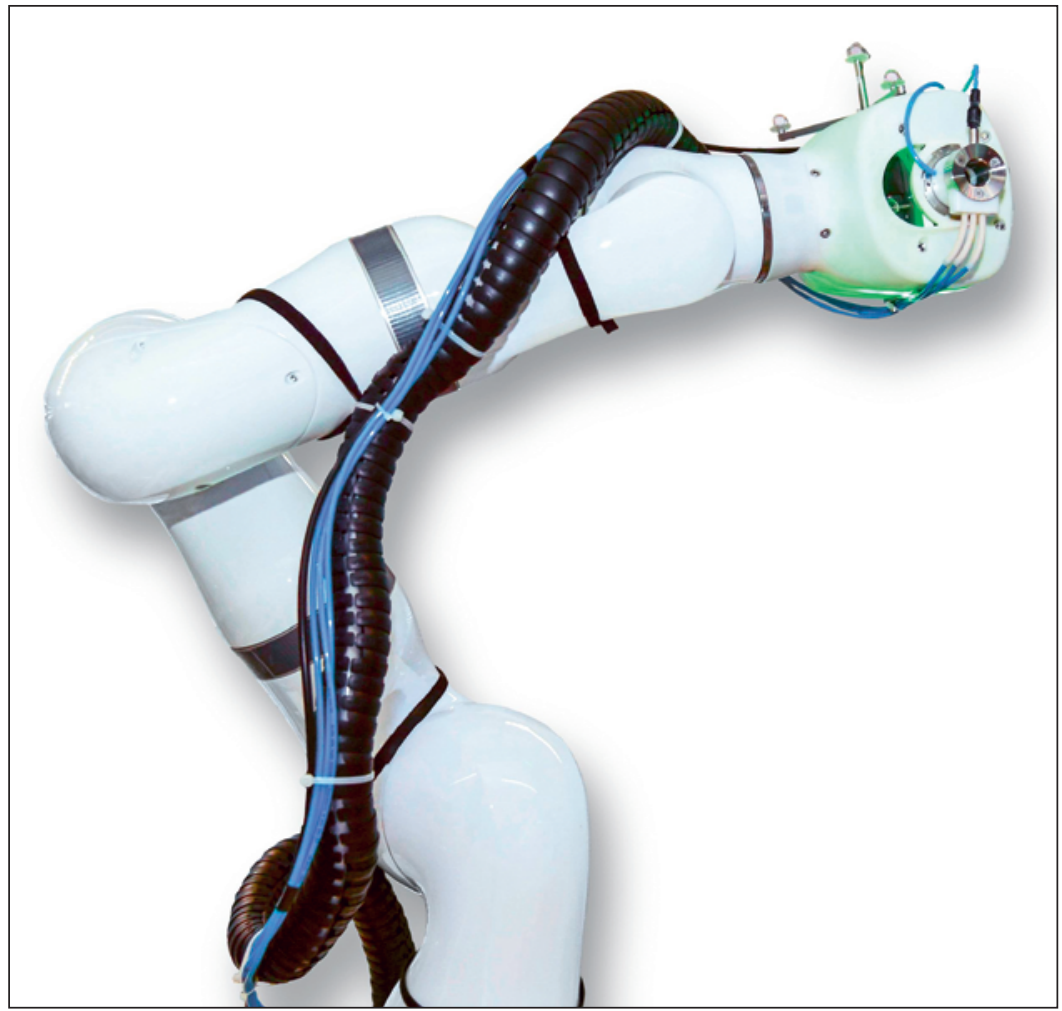

Operationsroboter Carlo kann Knochen berührungsfrei mit kalter Lasertechnologie schneiden. den dafür benötigten Experten spricht jeder seine eigene Fachsprache und hat seine eigene Wissens- und Forschungskultur entwickelt. Die eigentliche Herausforderung ist es, die unterschiedlichen Kulturen zusammenzubringen und eine gemeinsame Kultur zu entwickeln. Aus meiner Erfahrung kann ich sagen, dass Innovation oft genau an diesen Schnittstellen zwischen den Disziplinen entsteht. Um zurück auf die Roboter zu kommen: Ich bin überzeugt, dass gute Bedingungen und Guidelines im Umgang mit Robotern nur in dieser Dialog- oder Schnittstellenkultur entstehen kann.

\section{Der Roboter wird also den Arzt niemals ersetzen können?}

Nichts kann den menschlichen Kontakt zwischen Patient und Ärztin adäquat ersetzen. Die Empathie und die Kommunikation zwischen dem Patienten und dem Arzt kann kein Roboter ersetzen. Aber der Roboter kann ihn bei bestimmten Aufgaben ablösen, die er präziser und schneller erledigt. Wo beispielsweise spezielle geometrische Schnitte in der Chirurgie erforderlich sind, die der Chirurg händisch nicht durchführen kann.

Wir sollten uns hier von einem patientenzentrierten Vorgehen, einem "patient-centred approach», leiten lassen. Die Robotik wird sich immer dort durchsetzen, wo sie den Patienten Vorteile bringt. Der Arzt sollte sich fragen, was hilft dem Patienten, was kann der Roboter besser, und die Kompetenzen falls erforderlich abgeben. Künftig wird es zu einer Verlagerung der chirurgischen Tätigkeit weg vom handwerklich-chirurgischen zum planerisch-steuernden kommen.

\section{Wie gehen Sie mit Ängsten von Patienten um, die nicht von Robotern oder Maschinen abhängig sein möchten?}

In der Schweiz haben wir ein hervorragendes Gesundheitssystem, das höchste Qualität garantiert. Unsere Chirurgen haben eine sehr gute Ausbildung und jahrelange Erfahrung. Gleichzeitig haben wir strenge Kontrollmechanismen und hohe Qualitätsanforderungen.

\section{Nichts kann den menschlichen Kontakt zwi-} schen Patient und Ärztin adäquat ersetzen.

\section{untereinander: der Medizin und der Pflegewissen- schaft mit der Ethik, der Rechtswissenschaften, dem Ingenieurswesen und anderen. Warum, denken Sie, hat das noch nicht stattgefunden bisher?}

Sie plädieren für eine Vernetzung der Disziplinen

Wir haben bereits im Artikel dargestellt, wie wichtig diese Interdisziplinarität ist, denn Roboter im Operationssaal werfen viele Fragen auf: neben den medizinischen auch ethische, juristische und technische. Von
Wenn nun Roboter und Maschinen im operativen Umkönnen, dass die Qualität und Sicherheit nicht darunter leiden. Dann, da bin ich sicher, werden sich $\mathrm{Pa}$ tienten auch einem Roboter, unter der Führung eines guten, erfahrenen Chirurgen, anvertrauen. feld hinzukommen, muss der Patient darauf vertrauen 
Wie können wir sicherstellen, dass sich die Entwicklung von Robotern wirklich am Bedarf der Klinik, der Patienten und Ärztinnen, orientiert?

Dazu braucht es eine sinnvolle Zusammenarbeit zwischen den Ärzten, welche die Richtlinien erstellen, und den Organisationen, die die Patientensicherheit überwachen, sowie mit der Industrie, die marktorientiert arbeitet. Dafür benötigen wir entsprechende Forschungs- und Weiterentwicklungseinrichtungen an den Universitäten und den Spitälern. Wenn Industrie, Aufsichtsgremien und Wissenschaft zusammenarbeiten, dann können wir mit hoher Wahrscheinlichkeit davon ausgehen, dass das Endprodukt den Patientinnen und Patienten einen Nutzen bringt. Auch hier ist wieder Interdisziplinarität gefordert.

\section{Welche Kompetenzen benötigen Ärztinnen und Ärzte in Zukunft, um mit den Entwicklungen Schritt halten zu können?}

Es ist immens wichtig, dass wir die Kompetenzen für die Zukunft bereits heute vermitteln. Dabei müsste man gar nicht alles ins Grundstudium packen. Man

\section{"Wir werden bestimmte Jobs an Roboter abgeben, dafür aber andere neue benötigen und schaffen."}

wird vieles auch in postgraduierten Studiengängen lehren. Wir sollten mehr Mut zum Entrümpeln haben und vermehrt Neues in die Aus- und Weiterbildung aufnehmen. Wir bräuchten zum Beispiel schon heute einen Unterricht für Telemedizin, Virtuelle Realität oder Künstliche Intelligenz. Auch bräuchten wir Ärzte mehr spezifische Kenntnisse in Management, Leadership und Ethik, um nur einige zu nennen.

Im Bereich der digitalen Medizin und der Robotik brauchen wir sicherlich heute schon mehr Fachkräfte bei der Ärzteschaft, dem OP-Personal und der spezialisierten Pflege. Hier müssen wir Inhalte von komplexen Workflows, von der digitalen Bildgebung bis zur Bildverarbeitung, Planung, Navigation und der robotischen Umsetzung vermitteln. Aber auch bei der Weiterbildung von Fachleuten in der Industrie haben wir Nachholbedarf. Denn ein Industrieroboter ist nicht dasselbe wie ein Medizinroboter.

Um die Grundlage für diese Art von Wissensvermittlung zu schaffen, brauchen wir die Initiative von exzellenten universitären Spitälern, welche gemeinsam mit den Fachhochschulen, den Fachgesellschaften und der Industrie, die Ziele und Kompetenzen der zukünftigen Fachkräfte definieren.

\section{Müssen Ärzte wegen des Einzugs der Medizinroboter Angst um ihren Job haben?}

Der Arbeitsplatz der Zukunft wird anders aussehen als heute. Wir werden bestimmte Jobs an Roboter abgeben, dafür aber andere neue benötigen und schaffen. Diese Veränderungen und die damit verbundenen Unwägbarkeiten lösen existenzielle Ängste aus. Das ist bei Ärzten nicht anders als in anderen Berufen. Der einzelne Arzt mag sich angesichts der Veränderung ohnmächtig fühlen. Doch gemeinsam können wir Strukturen schaffen, welche uns für die künftigen Entwicklungen fit machen.

Ich plädiere hier auch an die gesellschaftliche Verantwortung, den Veränderungsprozess so zu gestalten, dass wir die Akteure in diesem Prozess begleiten, ohne die Innovationen zu bremsen, die wir für unsere $\mathrm{Zu}$ kunft so dringend benötigen. Wir müssen und wir wollen an der Spitze der Technologieentwicklung stehen, aber wir wollen dieser Technologie auch ein menschliches Gesicht geben.

Bildnachweis

Fotos: Universitätsspital Basel 